\section{Out of control}

\author{
Richard Rigby
}

$\mathrm{A}^{\mathrm{a}}$ er working in southern Africa for two months I still feel overwhelmed by the daily emotional and medical demands generated by the AIDS epidemic. I do not know whether my experience reflects that of other doctors, but perhaps an anecdotal report will convey the personal and professional impact of a disease better than a statistical presentation.

Considerations of HIV affect almost every decision of my working day-the male and female wards are continually filled with young, wasted adults with persistent coughs and other manifestations of tuberculosis, generalised lymphadenopathy, Kaposi's sarcoma, pyogenic infection, and neural and ocular manifestations of HIV infection. The main reason for admission is to exclude or identify treatable infections, especially pulmonary tuberculosis. The pressure on

\section{"Africa is a continent sitting on several time bombs.... The AIDS epidemic, however, is exploding today."}

beds demands early discharge for terminal and untreatable cases despite a hopelessly overstretched home support network. The horror at the magnitude of the problem is compounded by the inadequacy you feel when confronted by another patient deteriorating with a swinging temperature without the resources to investigate or treat the opportunistic infections associated with HIV. Every extra month of health salvaged from this disease is a success for the family and community as these are all parents and bread winners in a society where a hospital stay at planting or harvest time guarantees a malnourished family later in the year. Those deaths that do occur in hospital are most commonly caused by uncontrolled tuberculosis or by profuse diarrhoea combined with chronic wasting and anaemia. We have no side rooms and these lingering deaths occur on the open wards.

The children's ward is largely populated by babies and toddlers with a combination of pneumonia and failure to thrive. Persistent maternal antibodies make it difficult to say that they definitely have HIV infection, but those who respond to better nutrition and antibacterial or antituberculous treatment often return in a moribund state within weeks. In an environment with up to $40 \%$ vertical transmission rates, not only have some mothers lost several babies, but we also see many intrauterine deaths where HIV seems to be a causative agent.

The outpatient clinic today was another long shuffling queue of the same: clinic cards showing a steady decline in weight and successive courses of antibiotics; women presenting because of amenorrhoea due to dramatic weight loss; pathetic toddlers with horrendous neck nodes and a flat or declining growth curve; grandparents bringing their children's orphans; young men with the ominous scars of shingles. Leg oedema often reflects either marked inguinal lymphadenopathy or infiltration by Kaposi's sarcoma. Husband and wife are often admitted simultaneously. More traditional forms of sexually transmitted disease remain common and a large box of condoms resides on the doctor's desk for reinforcing the health education message to the commercial sex workers and their customers.

Meanwhile Africa's usual disasters continue apace: Hardlife dying from renal failure as a result of malaria and quinine; Cecilia unable to walk or talk following measles encephalitis; a maternal death from obstructed labour. And among all these are the daily bread of general practitioners everywhere-asthma, bronchitis, eczema, menorrhagia, and depression. But the pressure placed on beds, budgets, doctors, and community services by the HIV epidemic means that there are fewer resources for them now.

Denial is a feature of loss, trauma, and bereavement which can operate on a personal, local, national, and global scale. Many of our patients die in a state of denial. A whole language has evolved in society and the health services to avoid having to talk openly about AIDS. "N/S" or "new serology" becomes a euphemism for HIV, and "wasting syndrome" replaces AIDS even on death certificates. Sending a patient for "precounselling" is taken by patients and staff alike to mean that they are to have a HIV test, and to be "postcounselled" means that it was positive. Ordering a "check test" is a request for an inhouse, same day, HIV test. "TB 1 " is now used to distinguish typical, responsive pulmonary tuberculosis from "TB 2 ," which describes the patients with persistent cough, lymphadenopathy, weight loss, and a poor response to antituberculous treatment, who often die before their HIV results are available. This confusion over terminology presumably biases the death statistics where deaths from AIDS are hidden among the pneumonia, tuberculosis, and long illness or wasting syndrome.

Such language reflects the denial of nursing, medical, and laboratory staff who are worried about occupational and domestic exposure to HIV, and of authorities unable to cope with existing demands on health services. It also spares medical staff unable to deal with their impotence to treat the disease and relatives incapable of accepting their own vulnerability. Paradoxically, the other temptation is to assign every poorly understood symptom, unusual illness, or diagnostic dilemma to a manifestation of HIV. This response is a mechanism for coping with our inability to do anything further in such cases. HIV testing, however, often does render positive results in such cases.

Denial at some level is necessary for survival. If behind each case you felt the enormity of the personal, social, and psychological impact it would be unbearable. There is a 14 year old locally who looks after five siblings with no family support. Under this pressure even the extended African family is breaking down, through both fear and pure decimation. A hysterical paralysis was the presentation resulting from a second wife being evicted from the husband's home and then rejected by her parents after she was diagnosed as being HIV positive. Staff in hospitals and schools are continually on leave for the funerals of young relatives. Our hospital has two skilled members of staff whom we have been unable to replace. Where there is no denial there is despair: the defeated look on a wife's face as she hears her husband's diagnosis and fears for her own health and her children's future. One such

\section{"Deaths from AIDS are hidden among the pneumonia, tuberculosis, and long illness or wasting syndrome."}

woman recently demanded a church wedding to formalise their longstanding relationship as this would protect her inheritance from other frightened and jealous relatives when her husband died.

I am a new guest in this culture and I have much to learn about the concepts of illness and illness behaviour. The epidemic seems perpetuated not only by the denial prevalent within the illness response but also by cultural aspects of the relationship between men and women and particularly the relationship of power, authority, and wealth to sex. Teachers, soldiers, and police are particularly affected. As young skilled adults die they leave unfilled posts and unsupported families. The culture generates conflicting signals and messages for the schoolchildren. Posters, drama, and lessons encourage girls to say "No" and boys to wear condoms, but polygamy and prostitution are openly practised and accepted.

When I decided to work in Africa I believed I appreciated how bad the AIDS problem was. Now I do not believe anybody really does. Africa is a continent sitting on several time bombs: foreign debt, overpopulation, environmental degradation, and ethnic conflicts. The AIDS epidemic, however, is exploding today.-RICHARD RIGBY is a medical missionary in southern Africa 